\title{
Generalized Thermo Elasticity in an Infinite Nonhomogeneous Solid Having a Spherical Cavity Using DPL Model
}

\author{
Ahmed Elsayed Abouelrega \\ Department of Mathematics, Faculty of Science, Mansoura University, Mansoura, Egypt \\ E-mail:ahabogal@mans.edu.eg \\ Received January 2, 2011; revised March 39, 2011; accepted April 2, 2011
}

\begin{abstract}
The induced temperature, displacement, and stress fields in an infinite nonhomogeneous elastic medium having a spherical cavity are obtained in the context dual-phase-lag model. The surface of the cavity is stress free and is subjected to a thermal shock. The material is elastic and has an in homogeneity in the radial direction. The type of non homogeneity is such that the elastic constants, thermal conductivity and density are proportional to the nth power of the radial distance. The solutions are obtained analytically employing the Laplace transform technique. The numerical inversion of the transforms is carried out using Fourier series expansions. The stresses, temperature and displacement are computed and presented graphically. A comparison of the results for different theories is presented.
\end{abstract}

Keywords: Generalized Thermo Elasticity, Nonhomogeneous, Functionally Graded Material (FGM), Laplace Transform, Three-Phase-Lag Model

\section{Introduction}

The increasing use of anisotropic material in engineering application has resulted in considerable research activity in this area in recent years. An understanding of thermally induced stresses in an isotropic bodies is essential for a comprehensive study of their response due to an exposure to a temperature field, which may in turn occurs in service or during the manufacturing stages. For example, during the curing stages of lament wound bodies, thermal stresses may be induced from the heat buildup and cooling processes. The level of these stresses may be exceeding the ultimate strength.

The generalized thermoelasticity theories have been developed with the aim of removing the paradox of infinite speed of heat propagation inherent in the classical coupled dynamical thermo elasticity theory (C-D), see Biot [1]. Many new theories have been proposed to take care of this physical absurdity. Lord and Shulman [2] (L -S) first modified Fourier's law by introducing the term representing the thermal relaxation time. The heat equation associated this theory is a hyperbolic type and hence, eliminates the paradox of infinite speed of propagation. Following Green and Lindsay [3] (G-L) developed a more general theory of thermoelasticity, in which Fourier's law of heat conduction is unchanged, where as the classical energy equation and Duhamel-Neumann's relations are modified by introducing two constitutive constants having the dimensions of time.

Recently, relevant theoretical developments on this subject are due to Green and Naghdi [4] (G-N) to establish a theory of thermoelasticity that permits propagation of thermal waves at a finite speed, where its evolution equations are hyperbolic. An important characteristic feature of this theory, which is not present in other thermoelastic theories, is that this theory does not accommodate dissipation of thermal energy.

Tzou [5,6] proposed the dual-phase-lag (DPL) model, which describes the interactions between phonons and electrons on the microscopic level as retarding sources causing a delayed response on the macroscopic scale. For macroscopic formulation, it would be convenient to use the DPL mode for investigation of the micro-structural effect on the behavior of heat transfer. The physical meanings and the applicability of the DPL mode have been supported by the experimental results [7]. The dual-phase-lag (DPL) proposed by Tzou [7] is such a modification of the classical thermoelastic model in 
which the Fourier law is replaced by an approximation to a modified Fourier law with tow different time translations: a phase-lag of the heat flux $\tau_{q}$ and a phase-lag of temperature gradient $\tau_{\theta}$. A Taylor series approximation of the modified Fourier law, together with the remaining field equations leads to a complete system of equations describing a dual-phase-lag thermoelastic model. The model transmits thermoelastic disturbance in a wave-like manner if the approximation is linear with respect to $\tau_{q}$ and $\tau_{\theta}$, and $0 \leq \tau_{\theta}<\tau_{q}$; or quadratic in $\tau_{q}$ and linear in $\tau_{\theta}$, with $\tau_{q}>0$ and $\tau_{\theta}>0$. This theory is developed in a rational way to produce a fully consistent theory which is able to incorporate thermal pulse transmission in a very logical manner.

Each of models has been introduced in the literature in an attempt to eliminate shortcomings of the classical dynamical thermo elasticity such as: 1) infinite velocity of thermoelastic disturbances, 2) unsatisfactory thermoelastic response of a solid to short laser pulses, and 3) poor description of thermoelastic behavior at low temperature [17]. Attempts to present a theory of thermoelastic waves that would be attractive to both the basic and applied researchers have been continued in the literature to date. Also, a stream of papers devoted to theoretical and applied aspects of the generalized thermoelasticity that started with publications on the wave equation for a rigid heat conductor in the 1940s has increased greatly to date. Although the five theories are not the only ones that have been proposed so far, they are, in the authors opinion, representative in discussing the subject [17].

Allam, et. al. [11] investigated the thermal stress distributions in a harmonic field for a homogeneous, isotropic infinite body with a circular cylindrical hole based on a Green and Naghdi theory. Sinha and Elsibai [10] studied generalized thermoelastic interactions for an infinite body with a spherical cavity and for an isotropic solid sphere. Mukhopadhyay [12] discussed the thermally induced vibration in a homogeneous and isotropic unbounded body with a spherical cavity using the Green and Naghdi model of thermo elasticity without energy dissipation. Mukhopadhyay and Kumar [13] studied the thermoelastic interactions in an unbounded elastic medium with a spherical cavity in the context of four different theories of thermoelasticity, namely: the classical coupled dynamical thermoelasticity, the extended thermoelasticity, the temperature-rate-dependent thermoelasticity and the thermoelasticity without energy dissipation in a unified way. Roychoudhuri [14] studied one-dimensional thermoelastic wave propagation in an elastic halfspace in the context of dual-phase-lag mode.

Functionally graded materials (FGMs) are a type of nonhomogeneous materials in which the composition changes gradually with a corresponding change in the properties. FGMs are usually designed to be used under high temperature environments. Thermal shock loading conditions may be involved with high thermal stresses when a sudden heating or cooling happens. As a result, thermal fracture problems may be usually met. Therefore, it is significant to analyze the transient thermal fracture behavior of FGMs.

Functionally graded material (FGM) as a new kind of composites was initially designed as thermal barrier materials for aerospace structures, in which the volume fractions of different constituents of composites vary continuously from one side to another [15]. These novel nonhomogeneous materials have excellent thermo-mechanical properties to withstand high temperature and have extensive applications to important structures, such as aerospace, nuclear reactors, pressure vessels and pipes, chemicals plants, etc. The use of FGMs can eliminate or control thermal stresses in structural components [16].

Laplace transform technique is used to solve the problem. The main difficulty encountered in solving problems of coupled or generalized thermoelasticity theory is that of inversion of the Laplace transforms used. This is mainly due to the fact that the contour integral of Laplace transforms complex inversion formula contains complicated branch points in its integral. The inverse Laplace transforms are obtained numerically using a method based on Fourier expansion techniques.

The present paper is organized as follows. Section 2 describes the formulation of the problem and basic equations. Section 3 discusses the Laplace transform technique and the solution in the transformed domain is obtained using a potential function. Section 4 summarizes the inverse Laplace transforms using a numerical method based on Fourier expansion techniques. The last section is devoted to the numerical example for finding the temperature, displacement and the stress. The numerical results are presented graphically and compared it in the different theories of thermoelasticity and discussed the differences due to the presence of dual phase lags.

\section{Mathematical Formulation of the Problem and Basic Equations}

We consider a nonhomogeneous thermal and mechanical material properties in the radial direction infinite solid having a spherical cavity of radius a. The surface of the cavity is stress free and is suddenly heated and kept at constant temperature. We also assume that neither the body forces nor the heat sources are acting inside the medium.

We use spherical polar coordinates $(r, \theta, \phi)$ with the origin at the center of the cavity and we consider those thermoelastic interactions which are spherically symmetric. It follows that all interactions considered depend on 
the radial distance $r$ and the time $t$ then only the radial component of the displacement $u=u(r, t)$ and the normal stress components $\sigma_{r r}, \sigma_{\theta \theta}$ will appear in the analysis. The nonhomogeneous character of the material is discussed by considering that the elastic constants, density and the thermal conductivity are given by some power law of variation with radial distance $r$.

For a spherically orthotropic thermoelastic solid, the constitutive equations will take the following forms:

$$
\begin{aligned}
& \sigma_{r r}=C_{11}^{\prime} e_{r r}+C_{12}^{\prime}\left(e_{\theta \theta}+e_{\phi \phi}\right)-b_{1}^{\prime} T, \\
& \sigma_{\theta \theta}=C_{12}^{\prime} e_{r r}+C_{22}^{\prime} e_{\theta \theta}+C_{23}^{\prime} e_{\phi \phi}-b_{2}^{\prime} T
\end{aligned}
$$

where

$$
b_{1}^{\prime}=C_{11}^{\prime} \alpha_{1}+2 C_{12}^{\prime} \alpha_{2}, \quad b_{2}^{\prime}=C_{12}^{\prime} \alpha_{1}+C_{22}^{\prime} \alpha_{2}+C_{23}^{\prime} \alpha_{2}
$$

in which $C_{i j}$ are elastic constants, $T$ is the temperature excess over $T_{0}$, and $\alpha_{1}, \alpha_{2}$ are the coefficients of linear thermal expansion along the radial and tangential directions.

The equation of motion in absence of any body forces becomes

$$
\frac{\partial \sigma_{r r}}{\partial r}+\frac{2}{r}\left(\sigma_{r r}-\sigma_{\theta \theta}\right)=\rho \frac{\partial^{2} u}{\partial t^{2}}
$$

The non-zero components of strain are

$$
e_{r r}=\frac{\partial u}{\partial r}, e_{\theta \theta}=e_{\phi \phi}=\frac{u}{r}
$$

then the normal stresses are

$$
\begin{gathered}
\sigma_{r r}=C_{11}^{\prime} \frac{\partial u}{\partial r}+C_{12}^{\prime} \frac{2 u}{r}-b_{1}^{\prime} T, \\
\sigma_{\theta \theta}=C_{12}^{\prime} \frac{\partial u}{\partial r}+C_{22}^{\prime} \frac{u}{r}+C_{23}^{\prime} \frac{u}{r}-b_{2}^{\prime} T
\end{gathered}
$$

In an attempt to model ultrafast processes of thermoelasticity Tzou (1997) proposed a dual-phase-lag model (DPL) ofthermoelasticity in which the MaxwellCattaneo equation is replaced by the relation

$$
q+t_{q} q=-\nabla\left(K\left(T+t_{\theta} \dot{T}\right)\right), t_{q}>t_{\theta} \geq 0 .
$$

Equation (7) together with the energy balance equation led to the heat conduction equation

$$
\begin{aligned}
& \left(\frac{\partial}{\partial t}+t_{q} \frac{\partial^{2}}{\partial t^{2}}\right)\left[\rho C_{E} T+b_{1}^{\prime} T_{0}\left(\frac{\partial}{\partial r}+\frac{n+2}{r}\right) u\right] \\
& =\left(1+t_{\theta} \frac{\partial}{\partial t}\right)\left(\frac{1}{r^{2}} \frac{\partial}{\partial r}\right)\left[r^{2}\left(K_{r} \frac{\partial T}{\partial r}\right)\right]
\end{aligned}
$$

where $t_{q}$ and $t_{\theta}$ stand for the heat flux and temperature gradient phase-lags, respectively, $K_{r}$ is the is coefficient of thermal conductivity along radial direction, $C_{E}$ is specific heat at constant strain, $\rho$ is the density .

Clearly, a DPL model covers the hyperbolic L-S model when $t_{q}=t_{0}>0$, and $t_{\theta}=0$.

We assume that the functionally graded spherical that has nonhomogeneous thermal and mechanical properties in the radial direction. In order to incorporate the nonhomogeneity of the material, we assumed to take the following forms

$$
C_{i j}^{\prime}=r^{n} C_{i j}, \rho=r^{n} \rho_{0}, K_{r}=r^{n} K_{0},
$$

where $\rho_{0}, K_{0}$ and $C_{i j}$ are nonzero constants (they are the values of in a homogeneous matter when $n=0)$. Substituting from Equation (9) into Equations (5) and (6), we obtain

$$
\begin{gathered}
\sigma_{r r}=r^{n}\left(C_{11} \frac{\partial u}{\partial r}+C_{12} \frac{2 u}{r}-b_{1} T\right) \\
\sigma_{\theta \theta}=r^{n}\left(C_{12} \frac{\partial u}{\partial r}+\left(C_{22}+C_{23}\right) \frac{u}{r}-b_{2} T\right)
\end{gathered}
$$

where

$$
b_{1}=C_{11} \alpha_{1}+2 C_{12} \alpha_{2}, \quad b_{2}=C_{12} \alpha_{1}+\left(C_{22}+C_{23}\right) \alpha_{2}
$$

Using Equations (10) and (11), we have from Equation (3) the displacement formulation of the equation of motion

$$
\begin{gathered}
C_{11} \frac{\partial^{2} u}{\partial r^{2}}+C_{11} \frac{n+2}{r} \frac{\partial u}{\partial r}+2\left[(n+1) C_{12}-\left(C_{22}+C_{23}\right)\right] \frac{u}{r^{2}} \\
-b_{1} \frac{\partial T}{\partial r}-\left[(n+2) b_{1}-2 b_{2}\right] \frac{T}{r}=\rho_{0} \frac{\partial^{2} u}{\partial t^{2}} \\
\left(\frac{\partial}{\partial t}+t_{q} \frac{\partial^{2}}{\partial t^{2}}\right)\left[\rho_{0} C_{E} T+b_{1} T_{0}\left(\frac{\partial}{\partial r}+\frac{n+2}{r}\right) u\right] \\
=K_{0}\left(1+t_{\theta} \frac{\partial}{\partial t}\right)\left[\frac{\partial^{2} T}{\partial r^{2}}+\frac{n+2}{r} \frac{\partial T}{\partial r}\right]
\end{gathered}
$$

The problem is to solve Equations (12) and (13) subject to the boundary conditions

1) Traction-free cavity surface

$$
\sigma_{r r}(a, t)=0, t>0
$$

2) The surface of the cavity is considered to be maintained at a constant temperature To

$$
T(a, t)=T_{0}, t>0
$$

The initial and regularity conditions may be put as

$$
\begin{gathered}
T=\frac{\partial T}{\partial r}=u=\frac{\partial u}{\partial r}, t=0, a \leq r<\infty, \\
T(r, t)=0, u(r, t)=0, \text { at } r \rightarrow \infty
\end{gathered}
$$

The following non-dimensional quantities are introduced as 


$$
\begin{gathered}
r^{*}=\frac{r}{a}, \quad t^{*}=\frac{\eta}{a} t, \quad t_{i}^{*}=\frac{\eta}{a} \tau_{i},(i=q, \theta), \\
T^{*}=\frac{T}{T_{0}}, \quad u^{*}=\frac{C_{11}}{a T_{0} b_{1}} u, \quad \eta^{2}=\frac{C_{11}}{\rho}, \\
\sigma_{r r}^{*}=\frac{\sigma_{r r}}{r^{n} T_{0} b_{1}}, \sigma_{\theta \theta}^{*}=\frac{\sigma_{\theta \theta}}{r^{n} T_{0} b_{1}} .
\end{gathered}
$$

Using these non-dimensional variables, Equations (10)(13) take the form (dropping the asterisks for convenience):

$$
\begin{gathered}
\sigma_{r r}=r^{n}\left(\frac{\partial u}{\partial r}+\frac{C_{12}}{C_{11}} \frac{2 u}{r}-T\right) \\
\sigma_{\theta \theta}=r^{n}\left(\frac{\partial u}{\partial r}+\frac{\left(C_{22}+C_{23}\right)}{C_{12}} \frac{u}{r}-\frac{b_{2}}{b_{1}} T\right) \\
\frac{\partial^{2} u}{\partial r^{2}}+\frac{n+2}{r} \frac{\partial u}{\partial r}-m \frac{u}{r^{2}}-\frac{\partial^{2} u}{\partial t^{2}}=\frac{\partial T}{\partial r}+h \frac{T}{r} \\
\left(\frac{\partial}{\partial t}+t_{q} \frac{\partial^{2}}{\partial t^{2}}\right)\left[T+\varepsilon_{0}\left(\frac{\partial}{\partial r}+\frac{n+2}{r}\right) u\right] \\
=\chi\left(1+t_{\theta} \frac{\partial}{\partial t}\right)\left[\frac{\partial^{2} T}{\partial r^{2}}+\frac{n+2}{r} \frac{\partial T}{\partial r}\right]
\end{gathered}
$$

where

$$
\begin{gathered}
m=-\frac{2\left[(n+1) C_{12}-\left(C_{22}+C_{23}\right)\right]}{C_{11}}, h=\frac{\left[(n+2) b_{1}-2 b_{2}\right]}{b_{1}} \\
\varepsilon=\frac{b_{1}^{2} T_{0}}{\rho_{0} C_{E} \eta}, \chi=\frac{K_{0}}{a \rho_{0} C_{E} \eta}
\end{gathered}
$$

\section{Solution of the Problem}

Applying the Laplace transform to Equations (18)-(21), we get the field equations in the Laplace transform space as

$$
\begin{gathered}
\bar{\sigma}_{r r}=r^{n}\left(C_{11} \frac{\mathrm{d} \bar{u}}{\mathrm{~d} r}+C_{12} \frac{2 \bar{u}}{r}-b_{1} \bar{T}\right), \\
\bar{\sigma}_{\theta \theta}=r^{n}\left(\frac{\mathrm{d} \bar{u}}{\mathrm{~d} r}+\frac{\left(C_{22}+C_{23}\right)}{C_{12}} \frac{\bar{u}}{r}-\frac{b_{2}}{b_{1}} \bar{T}\right) \\
\frac{\mathrm{d}^{2} \bar{u}}{\mathrm{~d} r^{2}}+\frac{n+2}{r} \frac{\mathrm{d} \bar{u}}{\mathrm{~d} r}-\frac{n+2}{r} \frac{\bar{u}}{r}-s^{2} \bar{u}=\frac{\mathrm{d} \bar{T}}{\mathrm{~d} r}, \\
{\left[\frac{\mathrm{d}^{2}}{\mathrm{~d} r^{2}}+\frac{n+2}{r} \frac{\mathrm{d}}{\mathrm{d} r}-\omega^{2}\right] \bar{T}=\omega^{2} \varepsilon\left(\frac{\mathrm{d}}{\mathrm{d} r}+\frac{n+2}{r}\right) \bar{u}}
\end{gathered}
$$

where we assume $h=0, m=n+2$ and

$$
\omega^{2}=\frac{s\left(1+\tau_{q} s\right)}{\chi\left(1+\tau_{\theta} s\right)} \text {. }
$$

The boundary conditions in the Laplace transform space read

$$
\bar{T}(1, s)=\frac{1}{s}, \bar{\sigma}_{r r}(1, s)=0,
$$

while the regularity conditions are

$$
\bar{T}(r, s)=0, \bar{u}(r, s)=0 \text {, at } r \rightarrow \infty \text {. }
$$

Taking $\bar{u}=\frac{\mathrm{d} \bar{\phi}}{\mathrm{d} r}$, where $\bar{\phi}$ is the thermoelastic potential function and introducing it in Equations (24) and (25), we find

$$
\begin{gathered}
\left(D_{1} D_{2}-s^{2}\right) \bar{\phi}=\bar{T} \\
\left(D_{1} D_{2}-\omega^{2}\right) \bar{T}=\omega^{2} \varepsilon D_{1} D_{2} \bar{\phi}
\end{gathered}
$$

where

$$
D_{1}=\frac{\mathrm{d}}{\mathrm{d} r}+\frac{n+2}{r}, D_{2}=\frac{\mathrm{d}}{\mathrm{d} r}
$$

Eliminating $T$ from Equations (28) and (29), we get

$$
\left[\left(D_{1} D_{2}\right)^{2}-\left(\omega^{2}(1+\varepsilon)+s^{2}\right)\left(D_{1} D_{2}\right)+\omega^{2} s^{2}\right] \bar{\phi}=0
$$

Introducing $m_{i},(i=1,2)$ into Equation (30) become

$$
\left(D_{1} D_{2}-m_{1}^{2}\right)\left(D_{1} D_{2}-m_{2}^{2}\right) \bar{\phi}=0
$$

where $m_{i},(i=1,2)$ are the positive solutions of the following characteristic equation

$$
m^{2}-\left(\omega^{2}(1+\varepsilon)+s^{2}\right) m+\omega^{2} s^{2}=0
$$

In order to transform Equation (31) into a normal Bessell equation, a new dependant variable $\bar{\psi}(r)$ is introduced as

$$
\bar{\phi}=r^{-(n+1) / 2} \bar{\psi}(r)
$$

Then, Equation (31) is rewritten as

$$
\frac{\mathrm{d}^{2} \bar{\psi}}{\mathrm{d} r^{2}}+\frac{1}{r} \frac{\mathrm{d} \bar{\psi}}{\mathrm{d} r}-\left(\frac{v^{2}}{r^{2}}+m_{i}^{2}\right) \bar{\psi}=0, i=1,2,
$$

where

$$
v=\frac{n+1}{2}
$$

The solution of $\bar{\phi}$ in will be the form

$$
\bar{\phi}(r)=r^{-(n+1) / 2} \sum_{i=1}^{2}\left(A_{i} K_{v}\left(m_{i} r\right)+B_{i} I_{v}\left(m_{i} r\right)\right)
$$

where $K_{v}\left(m_{i} r\right)$ and $I_{v}\left(m_{i} r\right)$ are the modified Bessell functions of order $v$ of first and second kind respectively. $A_{1}$ and $A_{2}$ are independent of $r$ but depend on $S$ and are to be determined from the boundary conditions. In the case of spherical cavity the solution to be continuous every where, we take $B_{i}$ equal to zero. 
Using the relations between $\bar{u}(r), \bar{T}(r)$ and $\bar{\phi}(r)$, and using the recurrence relations of modified Bessell functions, we obtain the solution for the displacement $\bar{u}(r)$ and temperature $\bar{T}(r)$ as follows

$$
\begin{aligned}
& \bar{u}(r)= \\
& -\frac{r^{-(n+1) / 2}}{2 r} \sum_{i=1}^{2} A_{i}\left((n+1-2 v) K_{v}\left(m_{i} r\right)+2 m_{i} r K_{v+1}\left(m_{i} r\right)\right) \\
& \bar{T}(r)=r^{-(n+1) / 2} \sum_{i=1}^{2} A_{i}\left(m_{i}{ }^{2}-s^{2}\right) K_{v}\left(m_{i} r\right)
\end{aligned}
$$

Substituting the above solutions for $\bar{u}(r)$ and $\bar{T}(r)$ in the relations (22) and (23), we obtain the following solutions for $\bar{\sigma}_{r r}$ and $\bar{\sigma}_{\theta \theta}$ in the form

$$
\begin{aligned}
& \bar{\sigma}_{r r}=\frac{r^{-(n+1) / 2}}{2 r^{2} C_{11}} \sum_{i=1}^{2} A_{i}\left(L_{1} K_{v}\left(m_{i} r\right)-2 m_{i} r K_{v+1}\left(m_{i} r\right)\right) \\
& \bar{\sigma}_{\theta \theta}= \\
& -\frac{r^{-(n+1) / 2}}{4 r^{2} C_{11}} \sum_{i=1}^{2} A_{i}\left(\left(L_{5} m_{i}^{2}+L_{6}\right) K_{v}\left(m_{i} r\right)+4 m_{i} r L_{2} K_{v+1}\left(m_{i} r\right)\right)
\end{aligned}
$$

where

$$
\begin{aligned}
& L_{1}= \\
& \begin{array}{l}
C_{11}\left(n^{2}-2 n v+3 n-4 v+2+2 r^{2} s^{2}\right)+C_{12}(-2 n+4 v-2) \\
L_{2}=C_{11}(n+2)-2 C_{12}, L_{4}=-C_{12}(n+2)+2\left(C_{22}+C_{23}\right), \\
\quad L_{3}=C_{12}\left(-n^{2}+4 n v-3-4 n+8 v-4 v^{2}\right) \\
\quad+\left(C_{22}+C_{23}\right)(4 n-8 v+4) \\
L_{5}=4 r^{2} C_{11} \frac{b_{2}}{b_{1}}-4 C_{12} r^{2}, L_{5}=L_{3}-4 r^{2} C_{11} \frac{b_{2}}{b_{1}} s^{2} .
\end{array}
\end{aligned}
$$

In order to determine the parameters $A_{1}$ and $A_{2}$, we need to consider the boundary conditions (26), we get

$$
\begin{gathered}
\sum_{i=1}^{2} A_{i}\left(m_{i}^{2}-s^{2}\right) K_{v}\left(m_{i}\right)=\frac{1}{s}, \\
\sum_{i=1}^{2} A_{i}\left(L_{1} K_{v}\left(m_{i}\right)-2 m_{i} K_{v+1}\left(m_{i}\right)\right)=0
\end{gathered}
$$

Solving (39) and (40), we obtain $A_{1}$ and $A_{2}$ as the following

$$
\begin{aligned}
& A_{1}=\frac{1}{s \Delta}\left(L_{1} K_{v}\left(m_{2}\right)-2 m_{2} K_{v+1}\left(m_{2}\right)\right) \\
& A_{2}=\frac{-1}{s \Delta}\left(L_{1} K_{v}\left(m_{1}\right)-2 m_{1} K_{v+1}\left(m_{1}\right)\right)
\end{aligned}
$$

where

$$
\begin{aligned}
& \Delta=\left(m_{1}^{2}-s^{2}\right) K_{v}\left(m_{1}\right)\left(L_{1} K_{v}\left(m_{2}\right)-2 m_{2} K_{v+1}\left(m_{2}\right)\right) \\
& -\left(m_{2}^{2}-s^{2}\right) K_{v}\left(m_{2}\right)\left(L_{1} K_{v}\left(m_{1}\right)-2 m_{1} K_{v+1}\left(m_{1}\right)\right)
\end{aligned}
$$

Substituting the value of $A_{1}$ and $A_{2}$ from Equations (41) and (42) yields the displacement, temperature and thermal stresses in the transformed space.

The results for isotropic material can be deduced from our problem by simply replacing $C_{11}=\lambda+2 \mu, C_{12}=\lambda$, $C_{22}=C_{23}=\lambda+\mu, \quad b_{1}=b_{2}=(3 \lambda+2 \mu) \alpha_{t}$ and $K_{r}=K$ in our calculation, where $\lambda, \mu$ are the Lame's constants, $\alpha_{t}$ is the coefficient of linear thermal expansion and $K$ the thermal conductivity for the isotropic material.

\section{Inversion of the Laplace Transforms}

To obtain a solution of the problem in physical domain, we adopt a numerical inversion method based on a Fourier series expansion [9]. In this method, the inverse $g(t)$ of the Laplace transform $\bar{g}(\mathrm{~s})$ is approximated by the relation

$$
g(t)=\frac{\mathrm{e}^{c t}}{t_{1}}\left(\frac{\bar{g}(c)}{2}+\operatorname{Re} \sum_{k=1}^{N} \mathrm{e}^{i k t \pi / t_{1}} \bar{g}\left(c+\frac{i k \pi}{t_{1}}\right)\right), 0 \leq t \leq t_{1},
$$

where $N$ is a sufficiently large integer representing the number of terms in the truncated infinite Fourier series.

$N$ must chosen such that

$$
\mathrm{e}^{c t} \operatorname{Re}\left[\mathrm{e}^{i k t \pi / t_{1}} \bar{g}\left(c+\frac{i k \pi}{t_{1}}\right)\right] \leq \varepsilon_{1}
$$

where $\varepsilon_{1}$ is a persecuted small positive number that corresponds to the degree of accuracy to be achieved. The parameter $c$ is a positive free parameter that must be greater than the real parts of all singularities of $\bar{g}(s)$. Two methods are used to reduce the total error. First, the Korrecktur method is used to reduce the discretization error. Next, the algorithm is used to reduce the truncation error and therefore to accelerate convergence. The details of these methods can be found in Honig and Hirdes [9]. The discretization error can be made arbitrarily small by choosing the constant $\mathrm{c}$ large enough. The values of $c$ and $t_{1}$ are chosen according to the criteria outlined in [9]. Formula (43) was used to invert the Laplace transforms in Equations (35)-(38), given the temperature, stress, and displacement distribution numerically.

\section{Numerical Example and Discussion}

With the view of illustrating theoretical results obtained in the preceding sections, we now present some numerical results. The materials chosen for this purpose is single crystal of magnesium, the physical data for which is given 
as

$$
\begin{aligned}
C_{11} & =5.974 \times 10^{10} \mathrm{~N} \cdot \mathrm{m}^{-2}, C_{12}=2.624 \times 10^{10} \mathrm{~N} \cdot \mathrm{m}^{-2} \\
C_{23} & =2.17 \times 10^{10} \mathrm{~N} \cdot \mathrm{m}^{-2}, \\
C_{33} & =6.17 \times 10^{10} \mathrm{~N} \cdot \mathrm{m}^{-2}, \rho_{0}=1.47 \times 10^{3} \mathrm{~kg} \cdot \mathrm{m}^{-3}, \\
T_{0} & =298 \mathrm{~K}, \varepsilon=0.0202, \\
b_{1} & =b_{2}=2.68 \times 10^{6} \mathrm{Nm}^{-2} \mathrm{deg}^{-1} K_{0}=1.7 \times 10^{2}, \\
C_{\mathrm{E}} & =1.04 \times 10^{3}
\end{aligned}
$$

Numerical calculation are carried out for the temperature, the displacement, and the stress components along the $r$-direction. The computations were performed for one value of time, namely for $t=0.2$. For all numerical calculations Mathematica programming Language has been used. The results of the numerical evaluation of the thermoelastic stresses variations, displacement and temperature distribution are illustrated in Figures 1-8. The computational work has been performed for a sufficiently wide range of values of the nonhomogeneity index $n$, via $n=0,1,2,-1$ and -2 . Moreover $n=0$ leads to the case of a homogeneous medium.

Figures 1-4 elucidate the variation of dimensionless values of displacement, temperature, and thermal stresses distributions with respect to radius $r$ for different values of $n=0,1,2,-1$ and -2 . Figure 1 give the variation of the displacement with observation distance for different values of $n$, It is seen that displacement takes positive values and gradually increases until it attains a peak value at a particular location in close proximity to the cavity surface and then diminishes rapidly with increasing distance. Figure 2 represents the graph of temperature $T$ versus $r$. It is noted that near the region of the cavity, $T$ is maximum and it decreases with the increase of $r$, as it should occur in the real situation. Figure 3 represents the graph of radial thermal stress $\sigma_{r r}$ versus radius $r$. Also stress in each of the all cases is found to vanish on the boundaries, which agrees with the theoretical boundary condition. It is clear that for fixed $t$ it decreases with the increase of $r$, which is physically plausible. Figure 4 is plotted to show the variation of hoop stress $\sigma_{\theta \theta}$ versus $r$ for different values of $n$, from the graph it is clear that the maximum values of occur near the surface of the cavity and its magnitude decreases with the increase of $r$, keeping $t$ fixed.

Figures 5-8 show the displacement, temperature, radial stress and hoop stress distributions for three different values of the parameters $\tau_{\mathrm{q}}$ and $\tau_{\theta}$. These computations were carried out in the coupled theory $\left(\tau_{\mathrm{q}}=\tau_{\theta}=0\right)$, in Lord-Shulman theory $\left(\tau_{\theta}=0, \tau_{\mathrm{q}}=t_{0}>0\right)$ and in the generalized theory of thermoelasticity proposed by Tzou $\left(\tau_{\mathrm{q}}>\tau_{\theta} \geq 0\right)$. The numerical values of the temperature, displacement components and stress components are obtained and represented graphically for these theories.

\section{Conclusions}

The problem of investigating the thermoelastic stresses,

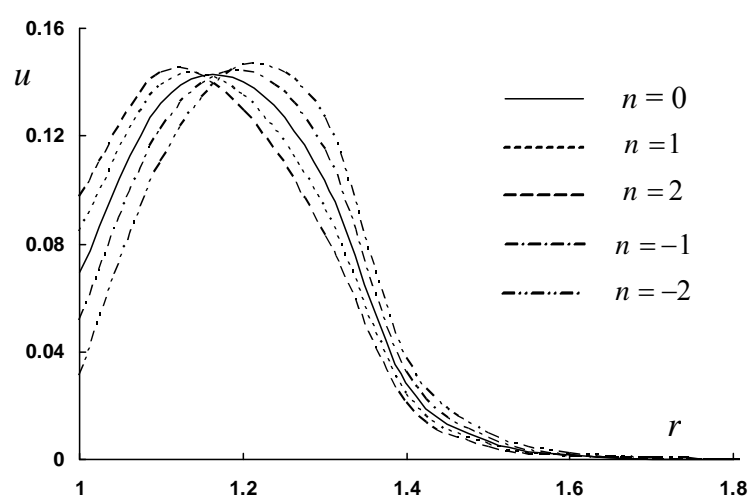

Figure 1. Variation of displacement with respect to radius for fifferent values of nonhomogeneity parameter $n$.

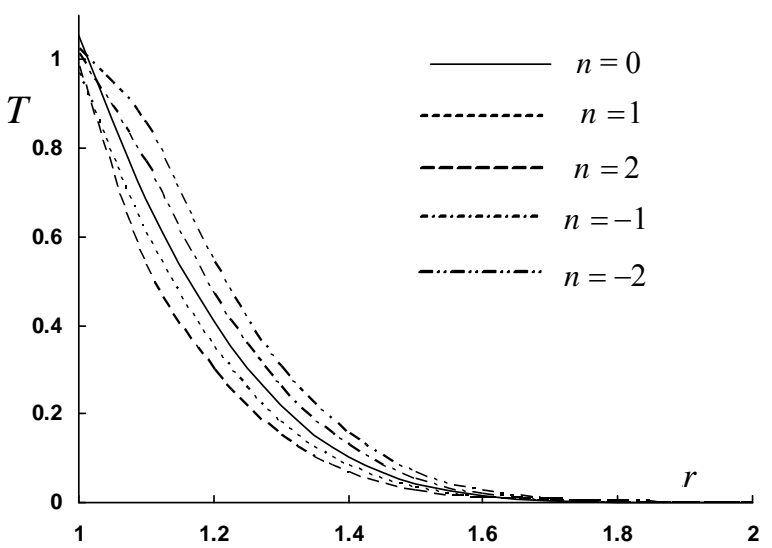

Figure 2. Variation of temperature with respect to radius for different values of nonhomogeneity parameter $n$.

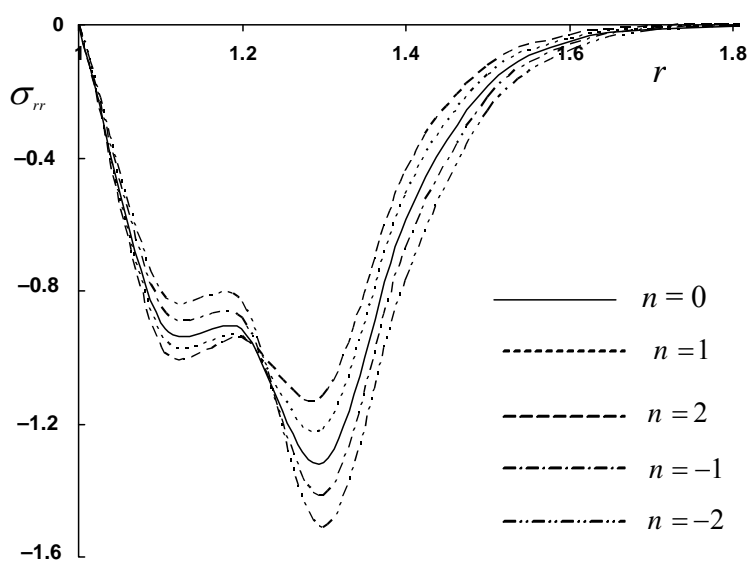

Figure 3. Variation of radial stress with respect to radius for different values of nonhomogeneity parameter $n$. 


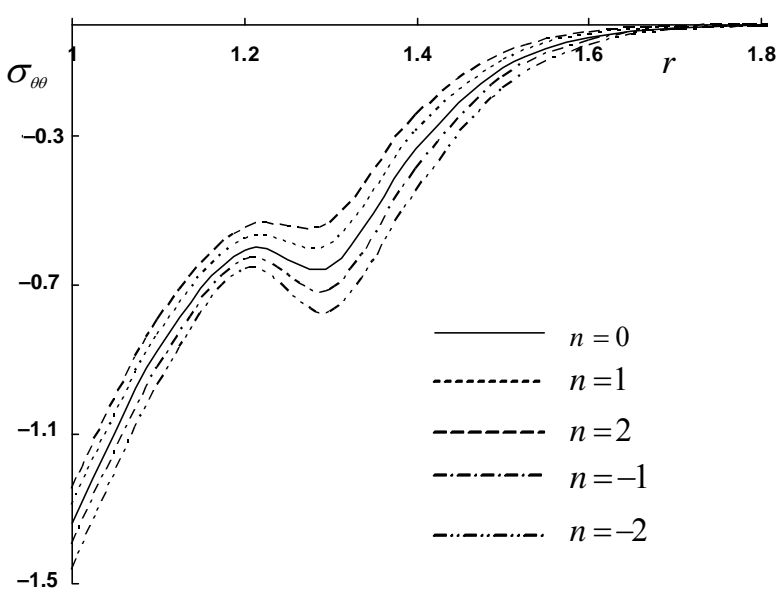

Figure 4. Variation of hoop stress with respect to radius for different values of nonhomogeneity parameter $n$.

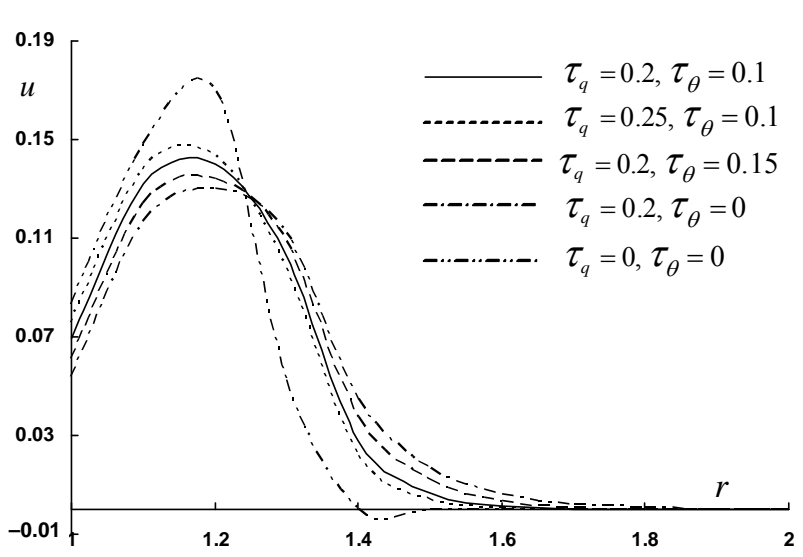

Figure 5. Variation of displacement with respect to radius for different values of phase-lags.

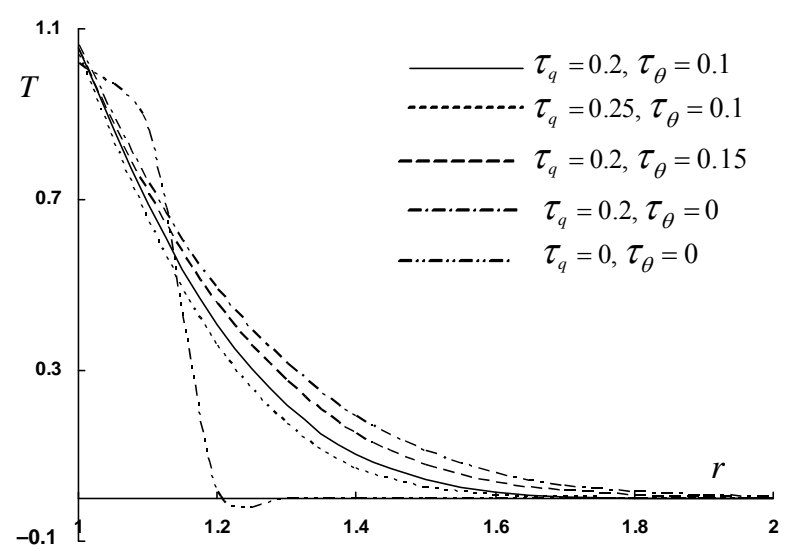

Figure 6. Variation of temperature with respect to radius for different values of phase-lags.

displacement and temperature distribution in a functionally graded orthotropic hollow sphere. Under thermal shock loading on the stress free boundaries of the hollow sphere the problem is studied using dual-phase-lag model

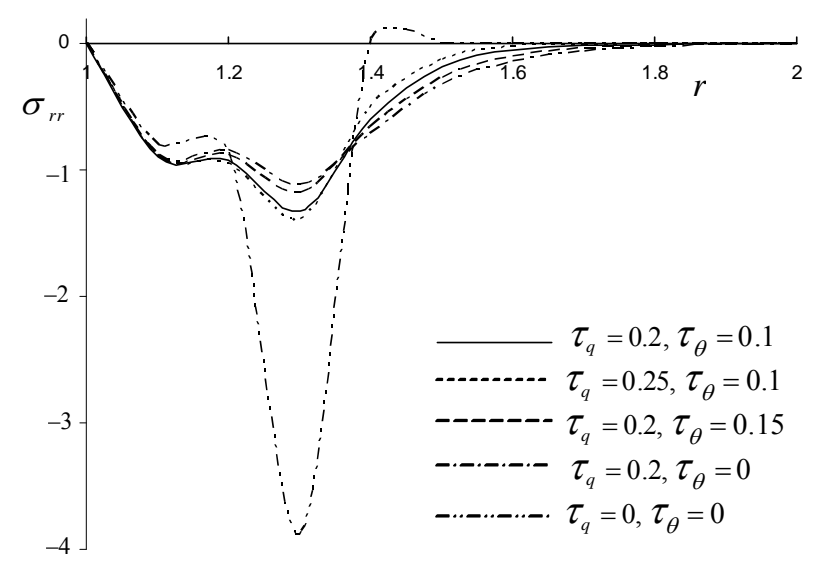

Figure 7. Variation of radial stress with respect to radius for different values of phase-lags.

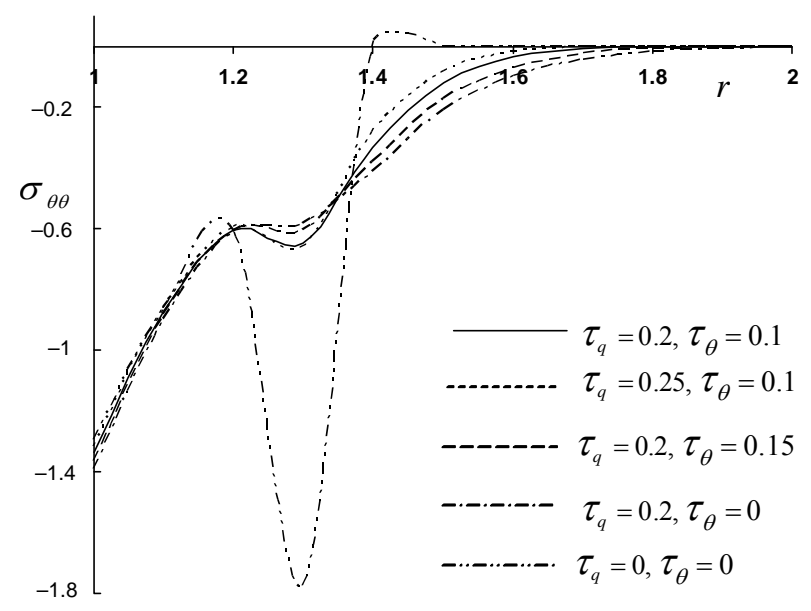

Figure 8. Variation of hoop stress with respect to radius for different values of phase-lags.

of generalized thermoelasticity. The thermophysical parameters are assumed to vary as the power-law exponent of the radial coordinate.

The analysis of the results permits some concluding remarks:

1) It can be observed that the phase lags effect plays a significant role on all distributions.

2) The difference between coupled theory and dualphase-lag (DPL) model is very clear.

3) The thermoelastic stresses, displacement and temperature have a strong dependency on the nonhomogeneous parameter n, so to design an FGM, the importance of the parameter must be taken into consideration.

4) The difference between Lord-Shulman theory (L-S), coupled theory and dual-phase-lag (DPL) model is rather small.

5) The phenomenon of finite speeds of propagation is manifested in all these figures. This is expected, since the thermal wave travels with a finite velocity. 


\section{References}

[1] M. A. Biot, "Thermoelasticity and Irreversible Thermodynamics," Journal of Applied Physics, Vol. 27, No. 3, 1956, pp. 240-253. doi:10.1063/1.1722351

[2] H. Lord and Y. Shulman, "A Generalized Dynamical Theory of Thermoelasticity," Journal of the Mechanics and Physics of Solids, Vol. 15, No. 5, 1967, pp. 299-309. doi:10.1016/0022-5096(67)90024-5

[3] A. E. Green and K. A. Lindsay, "Thermoelasticity," Journal of Elasticity, Vol. 2, No. 1, 1972, pp. 1-7. doi:10.1007/BF00045689

[4] A. E. Green and P. M. Naghdi, "Thermoelasticity Without Energy Dissipation," Journal of Elasticity, Vol. 31, No. 3, 1993, pp. 189-208. doi:10.1007/BF00044969

[5] D. Y. Tzou, "Macro- to Microscale Heat Transfer: The Lagging Behavior," 1st Edition, Taylor \& Francis, Washington, 1996.

[6] D. Y. Tzou, "A Unified Approach for Heat Conduction from Macro- to Microscales," Journal of Heat Transfer, Vol. 117, No. 1, 1995, pp. 8-16. doi:10.1115/1.2822329

[7] D. Y. Tzou, "Experimental Support for the Lagging Behavior in Heat Propagation," Journal of Thermophysics and Heat Transfer, Vol. 9, No. 4, 1995, pp. 686-693. doi: $10.2514 / 3.725$

[8] D. S. Chandrasekharaiah, "Thermoelasticity with Second Sound," Applied Mechanics Reviews, Vol. 39, No. 3, 1986, pp. 354-376.

[9] G. Honig and V. Hirdes, "A Method for the Numerical Inversion of the Laplace Transform," Journal of Computational and Applied Mathematics, Vol. 10, No. 1, 1984, pp. 113-132. doi:10.1016/0377-0427(84)90075-X

[10] S. B. Sinha and K. A. Elsibai, "Thermal Stresses for an
Infinite Body with Spherical Cavity with Tow Relaxation Times," Journal of Thermal Stresses, Vol. 19, No. 5, 1996, pp. 745-759. doi:10.1080/01495739608946190

[11] M. N. Allam, K. A. Elsibai and A. E. Abouelergal, "Thermal Stresses in a Harmonic Field for an Infinite Body with a Circular Cylindrical Hole Without Energy Dissipation," Journal of Thermal Stresses, Vol. 25, No. 1, 2002, pp. 57-68. doi:10.1080/014957302753305871

[12] S. Mukhopadhyay, "Thermoelastic Interactions without Energy Dissipation in an Unbounded Body with a Spherical Cavity Subjected to Harmonically Varying Temperature," Mechanics Research Communications, Vol. 31, No. 1, 2004, pp. 81-89.

doi:10.1016/S0093-6413(03)00082-X

[13] S. Mukhopadhyay and R. Kumar, "A Study of Generalized Thermoelastic Interactions in an Unbounded Medium with a Spherical Cavity," Computers and Mathematics with Applications, Vol. 56, No. 9, 2008, pp. 23292339.doi:10.1016/j.camwa.2008.05.031

[14] S. K. Roychoudhuri, "One-Dimensional Thermoelastic Waves in Elastic Half-Space with Dual-Phase-Lag Effects," Materials and Structures Journal, Vol. 2, No. 1, 2007, pp. 489-503. doi:10.2140/jomms.2007.2.489

[15] S. Suresh and A. Mortensen, "Fundamentals of Functionally Graded Materials," Institute of Materials Communications Ltd., London, 1998.

[16] R. C. Wetherhold and S. S. Wang, "The Use of Functionally Graded Materials to Eliminate or Control Thermal Deformation," Composites Science and Technology, Vol. 56, No. 9, 1996, pp. 1099-1104. doi:10.1016/0266-3538(96)00075-9

[17] R. B. Hetnarski and J. Ignaczak, "Generalized Thermoelasticity," Journal of Thermal Stresses, Vol. 22, No. 4, 1999, pp. 451-476. doi:10.1080/014957399280832 